group $(\mathrm{p}<0.01)$. 3. The level of LDH, CK, TNF- $\alpha$, IL-1 $\beta$, IL- 6 were no significant difference between Tet group and Sim group ( $p>0.05)$.

Conclusion Tet can attenuate myocardial ischaemia/reperfusion injury. It achieves this pharmacologic action through inhibition the I $\mathrm{B}-\alpha$ phosphorylation and reduces the harmful cytokine TNF- $\alpha$ and IL-6.

\section{e0130 TETRANDRINE CONTROL PRO-INFLAMMATORY FACTOR TO REDUCE RAT MYOCARDIAL ISCHAEMIC/REPERFUSION INJURY}

doi:10.1136/hrt.2010.208967.130

Yuqin Wang, Yuqin Wang. The Pla 252 hospital

Objective To investigate how tetrandrine through regulate the proinflammation factors TNF- $\alpha$, IL-1 $\beta$, IL- 6 to attenuate rat ischaemic/ reperfusion injury.

Methods Sprague-Dawley (SD) rats were randomly divided into four group: Sham, ischaemia/reperfusion (I/R), Tetrandrine (Tet) and simvastatin group (Sim). The SD rat underwent $30 \mathrm{~min}$ of left anterior descending (LAD) coronary occlusion and $24 \mathrm{~h}$ reperfusion to make ischaemia/reperfusion (I/R) injury model in vivo. Sham group were not subjected to occlusion of artery. Tet group were injected tetrandrine to abdominal cavity 20 min before ischaemia starting. The rat in Sim group was administrated simvastatin $2 \mathrm{mgkg} / \mathrm{l}$ intragastricly every day, administrating drugs lasted 14 days. The other procedures were same to the I/R group. Samples were collected after $24 \mathrm{~h}$ reperfusion. The expression level of TNF- $\alpha$, IL-1 $\beta$, IL- 6 protein in serum and myocardial tissue was detected by ELISA. LDH and CK were detected too. The neutrophil infiltration degree in myocardium was determined by using measuring the activity of myeloperoxidase (MPO) method. Cardiac function which includes $\mathrm{FS} \%, \mathrm{EF}$ and $\mathrm{E} / \mathrm{A}$ was measured by using ultrasound. EB/TTC (Azovan Blue/2, 3, 5-Tripheny-2H-Tetrazoliam Chloride) dyeing method was used to measure the infraction size.

Result 1 . The LDH and CK were significantly higher in I/R, Tet and Sim groups compared with Sham group $(p<0.01)$, but it were much lower in Tet and Sim groups compared with I/R group. 2. The cardiac function of systolic and dilator in experimental group was decreased significantly compared with normal heart's function. In Tet and Sim group, which was experienced pharmacological preconditioning their cardiac function were significant higher than I/ $\mathrm{R}$ group $(\mathrm{p}<0.01)$, but no significant difference between Tet and Sim on EF and E/A. 3. The activity of MPO was significantly increased after reperfusion, its activity in experimental groups were much higher than Sham group $(\mathrm{p}<0.01)$, notwithstanding its activity in Tet nad Sim groups were significantly lower than I/R group $(p<0.01)$. No significant difference was found between Tet and Sim group. 4. In Tet and Sim group the expression of proinflammatory factors (TNF- $\alpha$, IL-1 $\beta$, IL- 6 ) were significant lower compared with I/ R group $(p<0.01)$ and significant higher than shame group $(p<0.01)$. Conclusion Tet can attenuates myocardial ischaemia/reperfusion injury. It achieves this pharmacologic action through reduce the harmful cytokine TNF- $\alpha$ and IL- 6 , IL- $1 \beta$.

\section{e0131 THE ANTI-APOPTOTIC EFFECT OF INSULIN ON CARDIOCYTE IN DIABETIC RATS}

doi:10.1136/hrt.2010.208967.131

Liu Yaling, Cheng Xunmin, Jiang Shisen, Song Dan. Department of Cardiology, Clinic Medical School, Nanjing University /Nanjing General Hospital of Nanjing Military Command, Pla, Nanjing, China

Objective To observe the diverse apoptosis of the myocardiac mitochondria on insulin therapy in diabetic rats and to investigate the anti-apoptotic mechanism of insulin interacting with the mitochondria.

Methods Male wistar rats were administered with intraperitoneal injection of streptozotocin (STZ, $25 \mathrm{mg} / \mathrm{kg}$ ) and high fat diet to induce type 2 diabetic mellitus. Twenty-two were randomly divided into two treatment groups, namely, the early treatment group and the late treatment group (each $n=7$ ), and one diabetic (DM)group $(n=8)$. Another eight were chosen for control. Novolin 30R was administrated hypodermically to the early treatment group (IE group) at the first week and to the late treatment group (IL group) at the fourth week. DM group were injected subcutaneously with physiological saline. All groups were treated for 8 weeks. At the end of the experiment we compared SOD, MDA, GSH in different groups, as well as apoptotic index, mitochondrial membrane potential $\left(\Delta \Psi_{\mathrm{m}}\right)$, active oxygen and myocardial ultrastructure.

Results Compared to the control group, DM rats had higher blood glucose $(30.53 \pm 2.39$ vs $7.48 \pm 1.03, \mathrm{p}<0.01)$, HW/BW $(2.88 \pm 0.01$ vs $2.56 \pm 0.03, \mathrm{p}<0.05), \mathrm{MDA}(6.46 \pm 0.99$ vs $4.98 \pm 0.30, \mathrm{p}<0.01)$, apoptotic index $(0.934 \pm 0.032$ vs $0.063 \pm 0.011, \mathrm{p}<0.01)$, and active oxygen, but lower SOD $(222.06 \pm 12.94$ vs $245.99 \pm 8.67, \mathrm{p}<0.01)$, GSH $(6.99 \pm 1.50$ vs $9.71 \pm 0.67, \mathrm{p}<0.01)$ and $\Delta \Psi \mathrm{m}(0.243 \pm 0.087$ vs $0.900 \pm 0.075, p<0.01)$. The mitochondrial crista of DM rats break, dissolved and became vacuolous. Compared to the DM group, The level of MDA $(5.31 \pm 0.60$ vs $6.46 \pm 0.99, p<0.01)$ and apoptotic index $(0.48 \pm 0.07$ vs $0.93 \pm 0.03, p<0.01)$ were significantly lower and the level of $\Delta \Psi \mathrm{m}(0.63 \pm 0.09$ vs $0.24 \pm 0.09, \mathrm{p}<0.01)$ was increaseed in the IE group. The IE group showed remarkable improvement in contrast to the IL group which improved a little (MDA $(5.31 \pm 0.60$ vs $6.27 \pm 0.75, \mathrm{p}<0.01)$, apoptotic index $(0.48 \pm 0.07$ vs $0.90 \pm 0.03$, $\mathrm{p}<0.01), \Delta \Psi \mathrm{m}(0.63 \pm 0.09$ vs $0.35 \pm 0.04, \mathrm{p}<0.01)$.

Conclusion Insulin has an anti-apoptotic effect on cardiocytes of diabetic rats, and earlier intervention is better than later.

\section{e0132 MYOCARDIAL CAPILLARY PERICYTES IN RESPONSE TO HYPERTENSION WITH DIABETES}

doi:10.1136/hrt.2010.208967.132

Cheng Xunmin, Jiang Shisen, Wang Jun. Cardiology Department, Nanjing General Hospital of Nangjing Millitary Command of Pla, Nangjing, China

Introduction Pericytes are perivascular cells with multifunctional activities which are now being elucidated. Pericyte alteration or degeneration is linked directly with microangiopathy in diabetes, scleroderma and hypertension.

Aims The purpose of the present study is to investigate the pathologic changes of the myocardial capillary pericytes in hypertension with diabetes rats.

Methods The rat model of hypertensive with diabetes mellitus (SHDM) and the rat model of diabetes mellitus (DM) were induced by an intraperitoneal injection of streptozotocin combined with high fat diet in spontaneously hypertensive rats (SHR) and SD rats, respectively. The four groups were as follows: SD, DM, SHR and SHDM. The ultrastructure changes were examined by transmission electron microscope and the number of precity was assessed by immunohistochemistry of ventricular sections at 16 weeks.

Results Ultramicroscopic analysis of capillaries showed the pericytes on myocardial capillaries of SHR, DM, and SHDM were conspicuously abnormal in shape and were with cytoplasm containing abundant myofilament and organelle. In addition, pericytes seemed to be loosely associated with the endothelium. The number of pericytes in SHR, DM and SHDM were significantly increased than that in SD. The number of pericytes in SHDM were much higher than that in SHR $(11.8 \pm 3.6$ vs $3.9 \pm 1.1, p<0.01)$, but no significantly difference than that in DM $(11.8 \pm 3.6$ vs $10.2 \pm 3.3$, $\mathrm{p}>0.05)$. 